\title{
Eigenvalues of a Linear Transformation
}

\author{
Karol Pąk \\ Institute of Computer Science \\ University of Białystok \\ Poland
}

\begin{abstract}
Summary. The article presents well known facts about eigenvalues of linear transformation of a vector space (see [13]). I formalize main dependencies between eigenvalues and the diagram of the matrix of a linear transformation over a finite-dimensional vector space. Finally, I formalize the subspace $\bigcup_{i=0}^{\infty} \operatorname{Ker}(f-\lambda I)^{i}$ called a generalized eigenspace for the eigenvalue $\lambda$ and show its basic properties.
\end{abstract}

MML identifier: VECTSP11, version: $\underline{7.9 .03 \quad 4.108 .1028}$

The articles [11], [33], [2], [3], [12], [34], [8], [10], [9], [5], [31], [27], [15], [7], [14], [32], [35], [25], [30], [29], [28], [26], [6], [22], [16], [23], [20], [1], [19], [4], [21], [17], [18], and [24] provide the notation and terminology for this paper.

\section{Preliminaries}

We adopt the following convention: $i, j, m, n$ denote natural numbers, $K$ denotes a field, and $a$ denotes an element of $K$.

Next we state several propositions:

(1) Let $A, B$ be matrices over $K, n_{1}$ be an element of $\mathbb{N}^{n}$, and $m_{1}$ be an element of $\mathbb{N}^{m}$. If $\operatorname{rng} n_{1} \times \operatorname{rng} m_{1} \subseteq$ the indices of $A$, then $\operatorname{Segm}(A+$ $\left.B, n_{1}, m_{1}\right)=\operatorname{Segm}\left(A, n_{1}, m_{1}\right)+\operatorname{Segm}\left(B, n_{1}, m_{1}\right)$.

(2) For every without zero finite subset $P$ of $\mathbb{N}$ such that $P \subseteq \operatorname{Seg} n$ holds $\operatorname{Segm}\left(I_{K}^{n \times n}, P, P\right)=I_{K}^{\text {card } P \times \operatorname{card} P}$.

(3) Let $A, B$ be matrices over $K$ and $P, Q$ be without zero finite subsets of $\mathbb{N}$. If $P \times Q \subseteq$ the indices of $A$, then $\operatorname{Segm}(A+B, P, Q)=\operatorname{Segm}(A, P, Q)+$ $\operatorname{Segm}(B, P, Q)$. 
(4) For all square matrices $A, B$ over $K$ of dimension $n$ such that $i, j \in \operatorname{Seg} n$ holds Delete $(A+B, i, j)=\operatorname{Delete}(A, i, j)+\operatorname{Delete}(B, i, j)$.

(5) For every square matrix $A$ over $K$ of dimension $n$ such that $i, j \in \operatorname{Seg} n$ holds Delete $(a \cdot A, i, j)=a \cdot \operatorname{Delete}(A, i, j)$.

(6) If $i \in \operatorname{Seg} n$, then Delete $\left(I_{K}^{n \times n}, i, i\right)=I_{K}^{\left(n-^{\prime} 1\right) \times\left(n-^{\prime} 1\right)}$.

(7) Let $A, B$ be square matrices over $K$ of dimension $n$. Then there exists a polynomial $P$ of $K$ such that len $P \leq n+1$ and for every element $x$ of $K$ holds eval $(P, x)=\operatorname{Det}(A+x \cdot B)$.

(8) Let $A$ be a square matrix over $K$ of dimension $n$. Then there exists a polynomial $P$ of $K$ such that len $P=n+1$ and for every element $x$ of $K$ holds $\operatorname{eval}(P, x)=\operatorname{Det}\left(A+x \cdot I_{K}^{n \times n}\right)$.

Let us consider $K$. Observe that there exists a vector space over $K$ which is non trivial and finite dimensional.

\section{Maps With Eigenvalues}

Let $R$ be a non empty double loop structure, let $V$ be a non empty vector space structure over $R$, and let $I_{1}$ be a function from $V$ into $V$. We say that $I_{1}$ has eigenvalues if and only if:

(Def. 1) There exists a vector $v$ of $V$ and there exists a scalar $a$ of $R$ such that $v \neq 0_{V}$ and $I_{1}(v)=a \cdot v$.

For simplicity, we follow the rules: $V$ denotes a non trivial vector space over $K, V_{1}, V_{2}$ denote vector spaces over $K, f$ denotes a linear transformation from $V_{1}$ to $V_{1}, v, w$ denote vectors of $V, v_{1}$ denotes a vector of $V_{1}$, and $L$ denotes a scalar of $K$.

Let us consider $K, V$. One can verify that there exists a linear transformation from $V$ to $V$ which has eigenvalues.

Let $R$ be a non empty double loop structure, let $V$ be a non empty vector space structure over $R$, and let $f$ be a function from $V$ into $V$. Let us assume that $f$ has eigenvalues. An element of $R$ is called an eigenvalue of $f$ if:

(Def. 2) There exists a vector $v$ of $V$ such that $v \neq 0_{V}$ and $f(v)=$ it $\cdot v$.

Let $R$ be a non empty double loop structure, let $V$ be a non empty vector space structure over $R$, let $f$ be a function from $V$ into $V$, and let $L$ be a scalar of $R$. Let us assume that $f$ has eigenvalues and $L$ is an eigenvalue of $f$. A vector of $V$ is called an eigenvector of $f$ and $L$ if:

(Def. 3) $f($ it $)=L \cdot$ it.

We now state several propositions:

(9) Let given $a$. Suppose $a \neq 0_{K}$. Let $f$ be a function from $V$ into $V$ with eigenvalues and $L$ be an eigenvalue of $f$. Then

(i) $a \cdot f$ has eigenvalues, 
(ii) $a \cdot L$ is an eigenvalue of $a \cdot f$, and

(iii) $\quad w$ is an eigenvector of $f$ and $L$ iff $w$ is an eigenvector of $a \cdot f$ and $a \cdot L$.

(10) Let $f_{1}, f_{2}$ be functions from $V$ into $V$ with eigenvalues and $L_{1}, L_{2}$ be scalars of $K$. Suppose that

(i) $\quad L_{1}$ is an eigenvalue of $f_{1}$,

(ii) $\quad L_{2}$ is an eigenvalue of $f_{2}$, and

(iii) there exists $v$ such that $v$ is an eigenvector of $f_{1}$ and $L_{1}$ and an eigenvector of $f_{2}$ and $L_{2}$ and $v \neq 0_{V}$.

Then

(iv) $f_{1}+f_{2}$ has eigenvalues,

(v) $L_{1}+L_{2}$ is an eigenvalue of $f_{1}+f_{2}$, and

(vi) for every $w$ such that $w$ is an eigenvector of $f_{1}$ and $L_{1}$ and an eigenvector of $f_{2}$ and $L_{2}$ holds $w$ is an eigenvector of $f_{1}+f_{2}$ and $L_{1}+L_{2}$.

(11) $\mathrm{id}_{V}$ has eigenvalues and $\mathbf{1}_{K}$ is an eigenvalue of $\operatorname{id}_{V}$ and every $v$ is an eigenvector of $\mathrm{id}_{V}$ and $\mathbf{1}_{K}$.

(12) For every eigenvalue $L$ of $\operatorname{id}_{V}$ holds $L=\mathbf{1}_{K}$.

(13) If ker $f$ is non trivial, then $f$ has eigenvalues and $0_{K}$ is an eigenvalue of $f$.

(14) $f$ has eigenvalues and $L$ is an eigenvalue of $f$ iff $\operatorname{ker} f+(-L) \cdot \operatorname{id}_{\left(V_{1}\right)}$ is non trivial.

(15) Let $V_{1}$ be a finite dimensional vector space over $K, b_{1}, b_{1}^{\prime}$ be ordered bases of $V_{1}$, and $f$ be a linear transformation from $V_{1}$ to $V_{1}$. Then $f$ has eigenvalues and $L$ is an eigenvalue of $f$ if and only if Det AutEqMt $(f+$ $\left.(-L) \cdot \operatorname{id}_{\left(V_{1}\right)}, b_{1}, b_{1}^{\prime}\right)=0_{K}$.

(16) Let $K$ be an algebraic-closed field and $V_{1}$ be a non trivial finite dimensional vector space over $K$. Then every linear transformation from $V_{1}$ to $V_{1}$ has eigenvalues.

(17) Let given $f, L$. Suppose $f$ has eigenvalues and $L$ is an eigenvalue of $f$. Then $v_{1}$ is an eigenvector of $f$ and $L$ if and only if $v_{1} \in \operatorname{ker} f+(-L) \cdot \operatorname{id}_{\left(V_{1}\right)}$.

Let $S$ be a 1-sorted structure, let $F$ be a function from $S$ into $S$, and let $n$ be a natural number. The functor $F^{n}$ yields a function from $S$ into $S$ and is defined as follows:

(Def. 4) For every element $F^{\prime}$ of the semigroup of functions onto the carrier of $S$ such that $F^{\prime}=F$ holds $F^{n}=\prod\left(n \mapsto F^{\prime}\right)$.

In the sequel $S$ denotes a 1-sorted structure and $F$ denotes a function from $S$ into $S$.

Next we state several propositions:

(18) $F^{0}=\operatorname{id}_{S}$.

(19) $F^{1}=F$.

(20) $F^{i+j}=F^{i} \cdot F^{j}$. 
(21) For all elements $s_{1}, s_{2}$ of $S$ and for all $n, m$ such that $F^{m}\left(s_{1}\right)=s_{2}$ and $F^{n}\left(s_{2}\right)=s_{2}$ holds $F^{m+i \cdot n}\left(s_{1}\right)=s_{2}$.

(22) Let $K$ be an add-associative right zeroed right complementable Abelian associative well unital distributive non empty double loop structure, $V_{1}$ be an Abelian add-associative right zeroed right complementable vector space-like non empty vector space structure over $K, W$ be a subspace of $V_{1}, f$ be a function from $V_{1}$ into $V_{1}$, and $f_{3}$ be a function from $W$ into $W$. If $f_{3}=f\left\lceil W\right.$, then $f^{n} \uparrow W=f_{3}{ }^{n}$.

Let us consider $K, V_{1}$, let $f$ be a linear transformation from $V_{1}$ to $V_{1}$, and let $n$ be a natural number. Then $f^{n}$ is a linear transformation from $V_{1}$ to $V_{1}$.

We now state the proposition

(23) If $f^{i}\left(v_{1}\right)=0_{\left(V_{1}\right)}$, then $f^{i+j}\left(v_{1}\right)=0_{\left(V_{1}\right)}$.

\section{Generalized Eigenspace of a Linear Transformation}

Let us consider $K, V_{1}, f$. The functor UnionKers $f$ yielding a strict subspace of $V_{1}$ is defined by:

(Def. 5) The carrier of UnionKers $f=\left\{v ; v\right.$ ranges over vectors of $V_{1}: \bigvee_{n} f^{n}(v)=$ $\left.0_{\left(V_{1}\right)}\right\}$.

We now state a number of propositions:

(24) $v_{1} \in$ UnionKers $f$ iff there exists $n$ such that $f^{n}\left(v_{1}\right)=0_{\left(V_{1}\right)}$.

(25) $\operatorname{ker} f^{i}$ is a subspace of UnionKers $f$.

(26) $\operatorname{ker} f^{i}$ is a subspace of $\operatorname{ker} f^{i+j}$.

(27) Let $V$ be a finite dimensional vector space over $K$ and $f$ be a linear transformation from $V$ to $V$. Then there exists $n$ such that UnionKers $f=$ ker $f^{n}$.

(28) $f \nmid \operatorname{ker} f^{n}$ is a linear transformation from ker $f^{n}$ to ker $f^{n}$.

(29) $f\left\lceil\operatorname{ker}\left(f+L \cdot \operatorname{id}_{\left(V_{1}\right)}\right)^{n}\right.$ is a linear transformation from $\operatorname{ker}\left(f+L \cdot \operatorname{id}_{\left(V_{1}\right)}\right)^{n}$ to $\operatorname{ker}\left(f+L \cdot \operatorname{id}_{\left(V_{1}\right)}\right)^{n}$.

(30) $f\lceil$ UnionKers $f$ is a linear transformation from UnionKers $f$ to UnionKers $f$.

(31) $f\left\lceil\operatorname{UnionKers}\left(f+L \cdot \operatorname{id}_{\left(V_{1}\right)}\right)\right.$ is a linear transformation from $\operatorname{UnionKers}(f+$ $\left.L \cdot \operatorname{id}_{\left(V_{1}\right)}\right)$ to UnionKers $\left(f+L \cdot \operatorname{id}_{\left(V_{1}\right)}\right)$.

(32) $f\left\lceil\operatorname{im}\left(f^{n}\right)\right.$ is a linear transformation from $\operatorname{im}\left(f^{n}\right)$ to $\operatorname{im}\left(f^{n}\right)$.

(33) $f \nmid \operatorname{im}\left(\left(f+L \cdot \operatorname{id}_{\left(V_{1}\right)}\right)^{n}\right)$ is a linear transformation from $\operatorname{im}\left(\left(f+L \cdot \operatorname{id}_{\left(V_{1}\right)}\right)^{n}\right)$ to $\operatorname{im}\left(\left(f+L \cdot \operatorname{id}_{\left(V_{1}\right)}\right)^{n}\right)$.

(34) If UnionKers $f=\operatorname{ker} f^{n}$, then $\operatorname{ker} f^{n} \cap \operatorname{im}\left(f^{n}\right)=\mathbf{0}_{\left(V_{1}\right)}$. 
(35) Let $V$ be a finite dimensional vector space over $K, f$ be a linear transformation from $V$ to $V$, and given $n$. If UnionKers $f=\operatorname{ker} f^{n}$, then $V$ is the direct sum of ker $f^{n}$ and $\operatorname{im}\left(f^{n}\right)$.

(36) For every linear complement $I$ of UnionKers $f$ holds $f\lceil I$ is one-to-one.

(37) Let $I$ be a linear complement of $\operatorname{UnionKers}\left(f+(-L) \cdot \operatorname{id}_{\left(V_{1}\right)}\right)$ and $f_{4}$ be a linear transformation from $I$ to $I$. If $f_{4}=f\lceil I$, then for every vector $v$ of $I$ such that $f_{4}(v)=L \cdot v$ holds $v=0_{\left(V_{1}\right)}$.

(38) Suppose $n \geq 1$. Then there exists a linear transformation $h$ from $V_{1}$ to $V_{1}$ such that $\left(f+L \cdot \operatorname{id}_{\left(V_{1}\right)}\right)^{n}=f \cdot h+\left(L \cdot \operatorname{id}_{\left(V_{1}\right)}\right)^{n}$ and for every $i$ holds $f^{i} \cdot h=h \cdot f^{i}$.

(39) Let $L_{1}, L_{2}$ be scalars of $K$. Suppose $f$ has eigenvalues and $L_{1} \neq L_{2}$ and $L_{1}$ is an eigenvalue of $f$ and $L_{2}$ is an eigenvalue of $f$. Let $I$ be a linear complement of UnionKers $\left(f+\left(-L_{1}\right) \cdot \operatorname{id}_{\left(V_{1}\right)}\right)$ and $f_{4}$ be a linear transformation from $I$ to $I$. Suppose $f_{4}=f\left\lceil I\right.$. Then $f_{4}$ has eigenvalues and $L_{1}$ is not an eigenvalue of $f_{4}$ and $L_{2}$ is an eigenvalue of $f_{4}$ and UnionKers $\left(f+\left(-L_{2}\right) \cdot \operatorname{id}_{\left(V_{1}\right)}\right)$ is a subspace of $I$.

(40) Let $U$ be a finite subset of $V_{1}$. Suppose $U$ is linearly independent. Let $u$ be a vector of $V_{1}$. Suppose $u \in U$. Let $L$ be a linear combination of $U \backslash\{u\}$. Then $\overline{\bar{U}}=\overline{\overline{(U \backslash\{u\}) \cup\left\{u+\sum L\right\}}}$ and $(U \backslash\{u\}) \cup\left\{u+\sum L\right\}$ is linearly independent.

(41) Let $A$ be a subset of $V_{1}, L$ be a linear combination of $V_{2}$, and $f$ be a linear transformation from $V_{1}$ to $V_{2}$. Suppose the support of $L \subseteq f^{\circ} A$. Then there exists a linear combination $M$ of $A$ such that $f\left(\sum M\right)=\sum L$.

(42) Let $f$ be a linear transformation from $V_{1}$ to $V_{2}, A$ be a subset of $V_{1}$, and $B$ be a subset of $V_{2}$. If $f^{\circ} A=B$, then $f^{\circ}($ the carrier of $\operatorname{Lin}(A))=$ the carrier of $\operatorname{Lin}(B)$.

(43) Let $L$ be a linear combination of $V_{1}, F$ be a finite sequence of elements of $V_{1}$, and $f$ be a linear transformation from $V_{1}$ to $V_{2}$. Suppose $f \uparrow($ the support of $L) \cap \operatorname{rng} F$ ) is one-to-one and $\operatorname{rng} F \subseteq$ the support of $L$. Then there exists a linear combination $L_{3}$ of $V_{2}$ such that

(i) the support of $L_{3}=f^{\circ}(($ the support of $L) \cap \operatorname{rng} F)$,

(ii) $f \cdot(L F)=L_{3}(f \cdot F)$, and

(iii) for every $v_{1}$ such that $v_{1} \in($ the support of $L) \cap \operatorname{rng} F$ holds $L\left(v_{1}\right)=$ $L_{3}\left(f\left(v_{1}\right)\right)$.

(44) Let $A, B$ be subsets of $V_{1}$ and $L$ be a linear combination of $V_{1}$. Suppose the support of $L \subseteq A \cup B$ and $\sum L=0_{\left(V_{1}\right)}$. Let $f$ be a linear function from $V_{1}$ into $V_{2}$. Suppose $f \nmid B$ is one-to-one and $f^{\circ} B$ is a linearly independent subset of $V_{2}$ and $f^{\circ} A \subseteq\left\{0_{\left(V_{2}\right)}\right\}$. Then the support of $L \subseteq A$. 


\section{REFERENCES}

[1] Jesse Alama. The rank+nullity theorem. Formalized Mathematics, 15(3):137-142, 2007.

[2] Grzegorz Bancerek. Cardinal numbers. Formalized Mathematics, 1(2):377-382, 1990.

[3] Grzegorz Bancerek. The fundamental properties of natural numbers. Formalized Mathematics, 1(1):41-46, 1990.

[4] Grzegorz Bancerek. Monoids. Formalized Mathematics, 3(2):213-225, 1992.

[5] Grzegorz Bancerek and Krzysztof Hryniewiecki. Segments of natural numbers and finite sequences. Formalized Mathematics, 1(1):107-114, 1990.

[6] Czesław Byliński. Binary operations applied to finite sequences. Formalized Mathematics, 1(4):643-649, 1990.

[7] Czesław Byliński. Finite sequences and tuples of elements of a non-empty sets. Formalized Mathematics, 1(3):529-536, 1990.

[8] Czesław Byliński. Functions and their basic properties. Formalized Mathematics, 1(1):5565, 1990.

[9] Czesław Byliński. Functions from a set to a set. Formalized Mathematics, 1(1):153-164, 1990.

[10] Czesław Byliński. Partial functions. Formalized Mathematics, 1(2):357-367, 1990.

[11] Czesław Byliński. Some basic properties of sets. Formalized Mathematics, 1(1):47-53, 1990.

[12] Agata Darmochwał. Finite sets. Formalized Mathematics, 1(1):165-167, 1990.

[13] I.N. Herstein and David J. Winter. Matrix Theory and Linear Algebra. Macmillan, 1988.

[14] Katarzyna Jankowska. Matrices. Abelian group of matrices. Formalized Mathematics, 2(4):475-480, 1991.

[15] Eugeniusz Kusak, Wojciech Leończuk, and Michał Muzalewski. Abelian groups, fields and vector spaces. Formalized Mathematics, 1(2):335-342, 1990.

[16] Robert Milewski. Associated matrix of linear map. Formalized Mathematics, 5(3):339$345,1996$.

[17] Robert Milewski. The evaluation of polynomials. Formalized Mathematics, 9(2):391-395, 2001.

[18] Robert Milewski. Fundamental theorem of algebra. Formalized Mathematics, 9(3):461470, 2001.

[19] Robert Milewski. The ring of polynomials. Formalized Mathematics, 9(2):339-346, 2001.

[20] Michał Muzalewski. Rings and modules - part II. Formalized Mathematics, 2(4):579-585, 1991.

[21] Michał Muzalewski and Lesław W. Szczerba. Construction of finite sequences over ring and left-, right-, and bi-modules over a ring. Formalized Mathematics, 2(1):97-104, 1991.

[22] Karol Pąk. Basic properties of the rank of matrices over a field. Formalized Mathematics, 15(4):199-211, 2007.

[23] Karol Pa̧k and Andrzej Trybulec. Laplace expansion. Formalized Mathematics, 15(3):143$150,2007$.

[24] Karol Pąk. Linear map of matrices. Formalized Mathematics, 16(3):269-275, 2008.

[25] Andrzej Trybulec. Domains and their Cartesian products. Formalized Mathematics, 1(1):115-122, 1990.

[26] Wojciech A. Trybulec. Basis of vector space. Formalized Mathematics, 1(5):883-885, 1990.

[27] Wojciech A. Trybulec. Groups. Formalized Mathematics, 1(5):821-827, 1990.

[28] Wojciech A. Trybulec. Linear combinations in vector space. Formalized Mathematics, $1(5): 877-882,1990$.

[29] Wojciech A. Trybulec. Operations on subspaces in vector space. Formalized Mathematics, 1(5):871-876, 1990.

[30] Wojciech A. Trybulec. Subspaces and cosets of subspaces in vector space. Formalized Mathematics, 1(5):865-870, 1990.

[31] Wojciech A. Trybulec. Vectors in real linear space. Formalized Mathematics, 1(2):291-296, 1990.

[32] Wojciech A. Trybulec. Lattice of subgroups of a group. Frattini subgroup. Formalized Mathematics, 2(1):41-47, 1991.

[33] Zinaida Trybulec. Properties of subsets. Formalized Mathematics, 1(1):67-71, 1990.

[34] Edmund Woronowicz. Relations and their basic properties. Formalized Mathematics, 1(1):73-83, 1990. 
[35] Katarzyna Zawadzka. The product and the determinant of matrices with entries in a field. Formalized Mathematics, 4(1):1-8, 1993.

Received July 11, 2008 